\title{
Hyperbaric prilocaïne for one-day spinal anesthesia: a large cohort retrospective study
}

\author{
Andlauer J.A. ${ }^{1}$, Ngatcha R. ${ }^{1}$, Gouwy J. ${ }^{1}$, Renard M. ${ }^{1}$, Ifrim D. ${ }^{1}$, Kapessidou Y.2, Guntz E. \\ ${ }^{1}$ Hôpital de Braine l'Alleud-Waterloo, Service d'Anesthésiologie - Braine l'Alleud (Belgique); \\ ${ }^{2} \mathrm{CHU}$ Saint-Pierre, Service d'Anesthésiologie-Réanimation - Bruxelles (Belgique)
}

\section{INTRODUCTION}

Hyperbaric $2 \%$ prilocaine (HP) is increasingly used for spinal anesthesia. Therefore, we conducted a retrospective study on a large panel of patients scheduled for day-case surgeries. Intrathecal HP was evaluated in terms of efficiency, side effects, complications and also the effect of sufentanil as an adjuvant.

\section{MATERIAL AND METHODS}

Following data were collected: failure, hypotension-bradycardia, nausea, urinary retention, mean satisfaction score (graded from 0 to 10), mean first VAS during PACU stay and mean maximum VAS (0-10), TNS. Intrathecal HP was compared to the association HP-sufentanil, by using a propensity score, after matching groups on the following confounding variables: gender, age, weight, size, BMI, ASA score, type of surgery, dose of HP, analgesics, midazolam.

\section{RESULTS AND DISCUSSION}

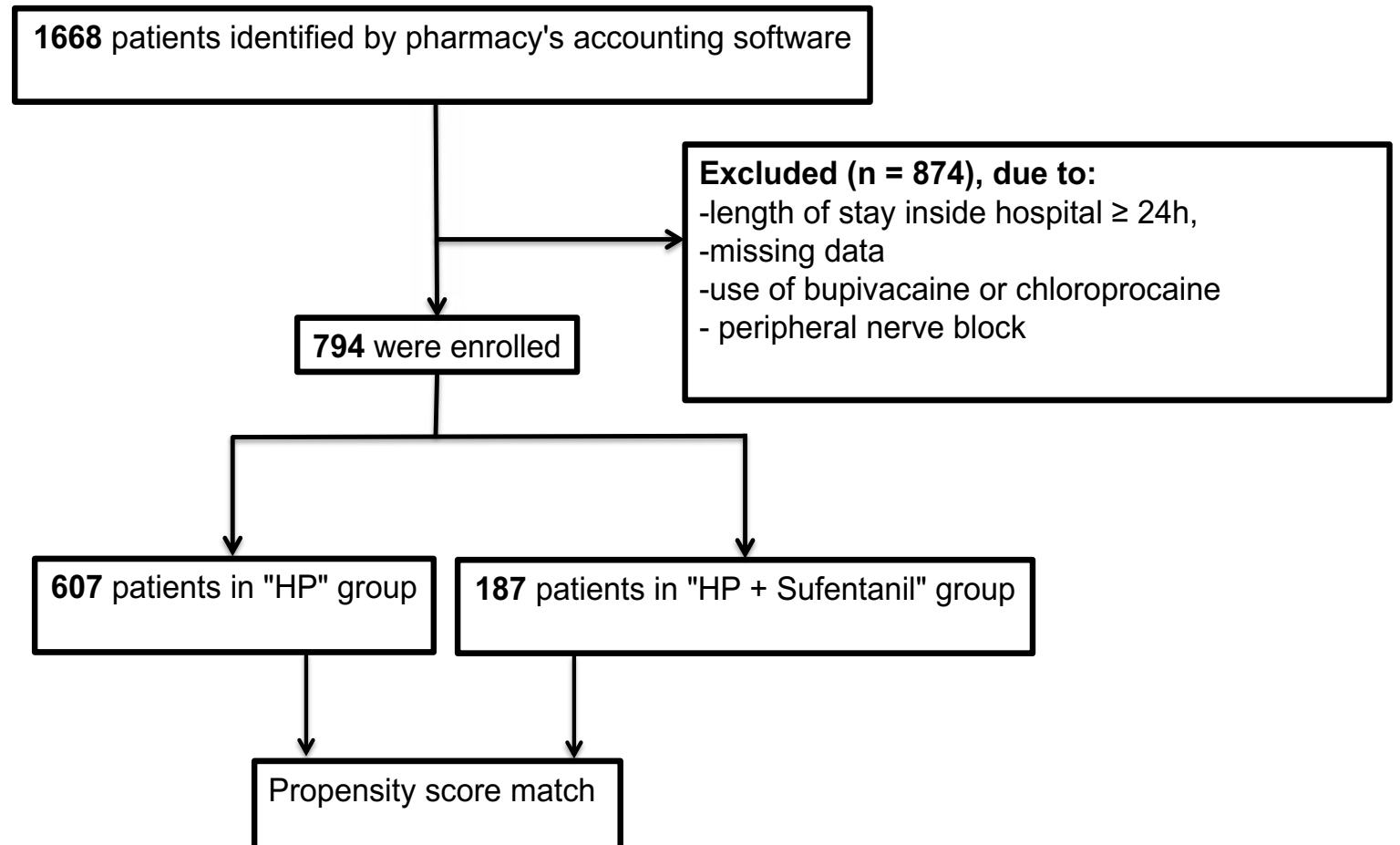

Fig 1: Flowchart
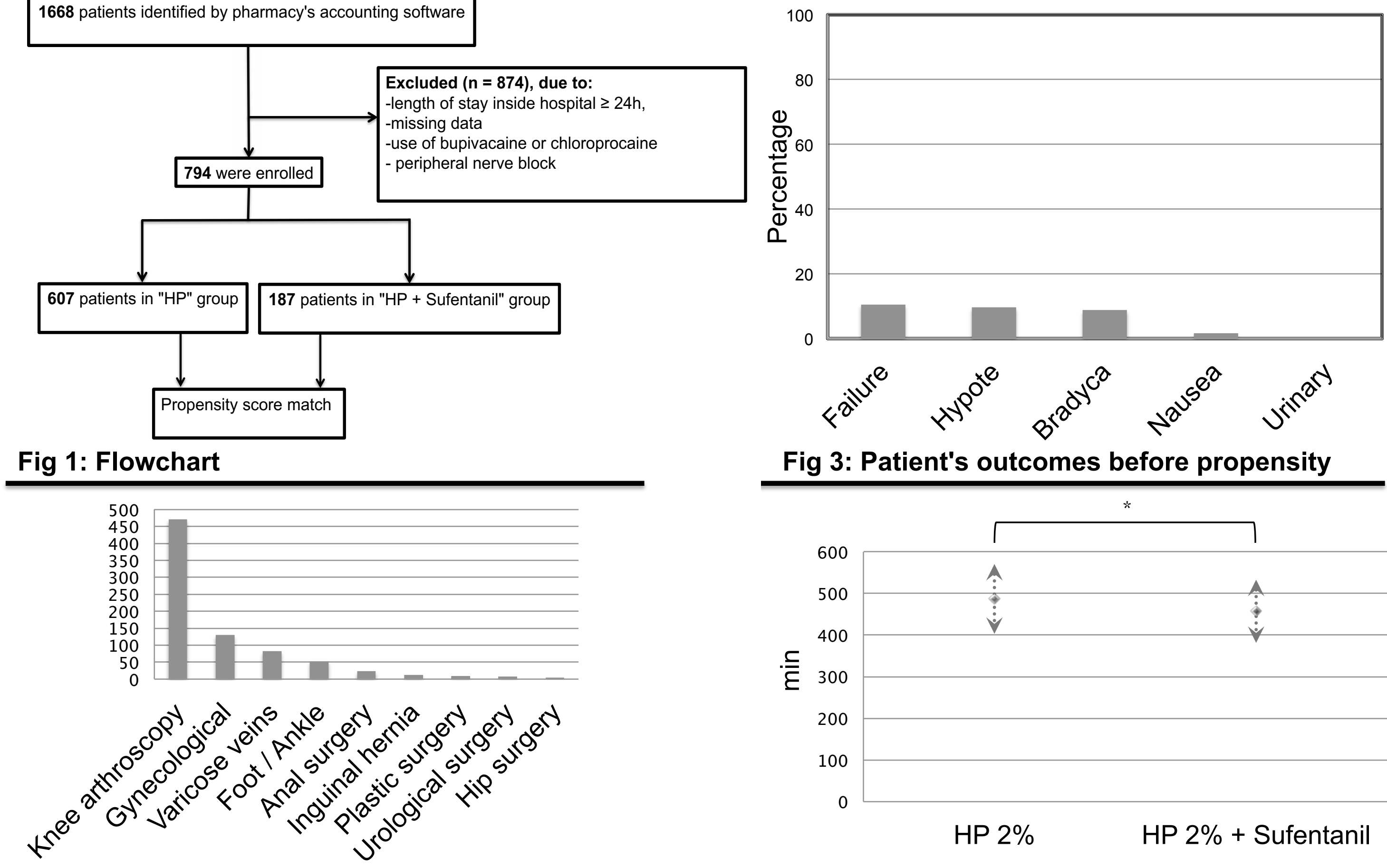

Fig 3: Patient's outcomes before propensity

Fig 2: Distribution of surgeries

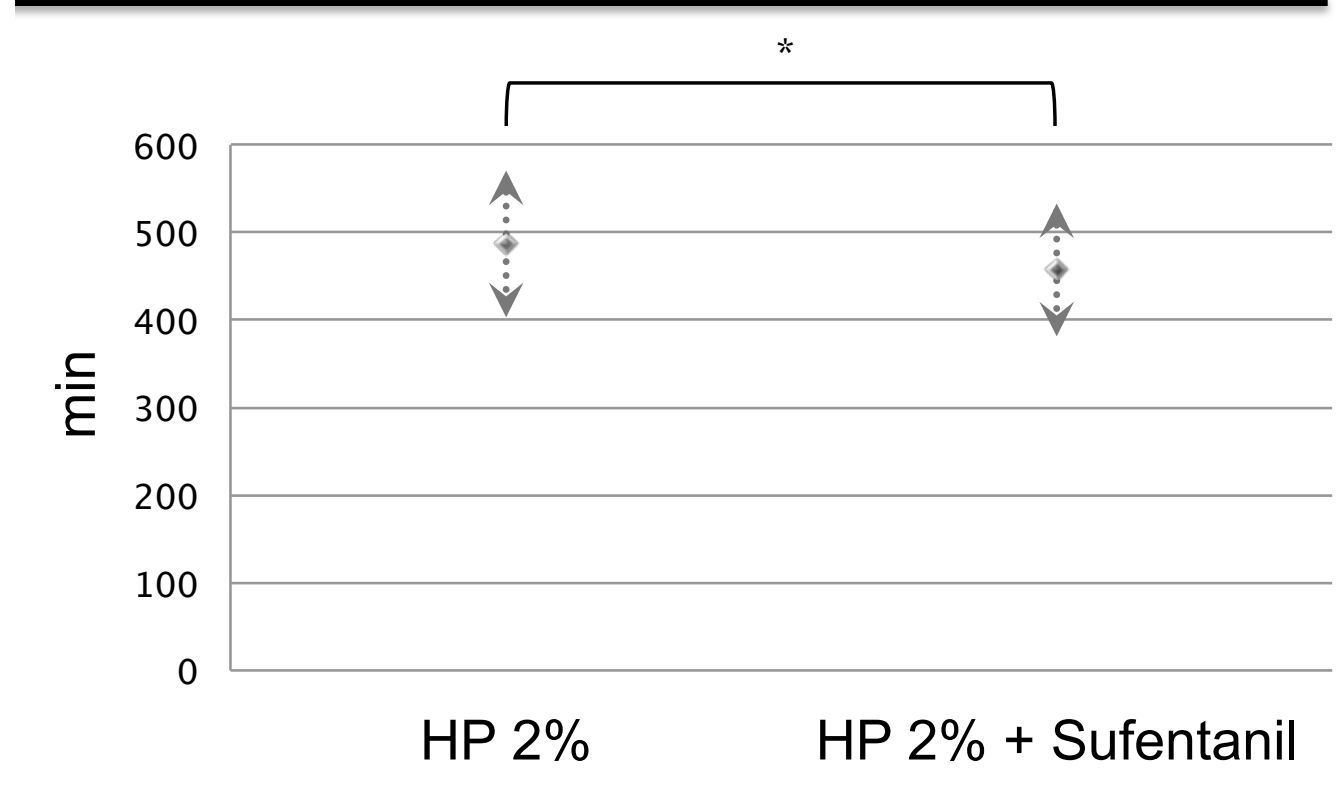

Fig 4: Length of stay

- Mean satisfaction score: $8.51 \pm 1.87$

- Mean first recorded VAS in the PACU: 0.11 [0-9]

One patient described typical Clinical signs of TNS

- Mean maximum VAS: 0.76 [0-9] 